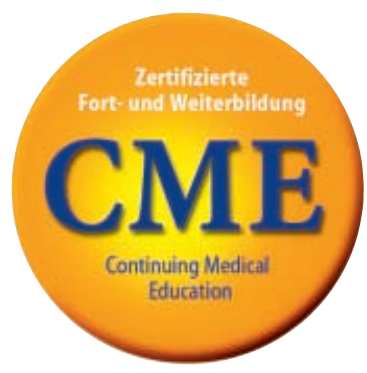

CME steht als Abkürzung für «continuing medical education» bzw. kontinuierliche medizinische Fortbildung, was nichts anderes bedeutet, als dass Fortbildung von Ärzten, die ja immer schon stattfand, nun auch dokumentierbar sein soll-auffreiwilliger Basis durch ein freiwilliges Fortbildungszertifikat.

Die Fortbildungszertifizierung ist 1999 durch den Deutschen Ärztetag bundesweit empfohlen worden. Im September 2000 hat die Bundesärztekammer einheitliche Bewertungskriterien für das freiwillige Fortbildungszertifikat herausgegeben (s. u.).

Im November 2000 hat der SpringerVerlag begonnen, die Rubrik Fort- und Weiterbildung in den Facharztzeitschriften in ein Zertifizierungsmodell einzugliedern. Mittlerweile konnten wir das Angebot auf die meisten Fachgebiete der Medizin erweitern.

Einheitliche Bewertungskriterien für das freiwillige Fortbildungszertifikat

Nach den einheitlichen Bewertungskriterien für das freiwillige Fortbildungszertifikat kann im Zuständigkeitsbereich der meisten Landesärztekammern ein freiwilliges Fortbildungszertifikat (150 Fortbildungspunkte in 3 Jahren) erworben werden. Maximal 30 dieser Punkte (10 Fortbildungspunkte/Jahr) können durch strukturierte interaktive Fortbildung gesammelt werden, so $z$. B. durch die Beantwortung der Fragebögen in dieser Facharztzeitschrift.

\title{
Module zur zertifizierten Fort- und Weiterbildung in den Springer-Facharztzeitschriften
}

\section{Mitmachen und bei den \\ Landesärztekammern \\ Fortbildungspunkte sammeln}

Wir möchten Sie ermuntern, die Fragebögen auszufüllen und an uns zurückzufaxen. In den Monaten Dezember und Juni erhalten Sie von uns per Fax die Auswertungen Ihrer Fragebögen mit Ihrem prozentualen Ergebnis. Die korrekten Antworten können Sie auch in der übernächsten Ausgabe Ihrer Zeitschrift erfahren.

Als Besonderheit bieten wir Ihnen an, alles auch bequem über das Internet zu erledigen unter www.medizinonline.de. Als Service können Sie hier Ihre Ergebnisse nach Ablauf der Sperrfrist jederzeit online abfragen.

Wir bitten Sie, Ihre Auswertungsbögen aufzubewahren und diese dann zusammen mit Ihren anderen Unterlagen bei Ihrer Ärztekammer zum Erwerb des freiwilligen Fortbildungszertifikats einzureichen, wenn die Voraussetzungen erfüllt sind. Bei Bedarf informiert Sie Ihre Landesärztekammer, ob und ggf. wie der Erwerb eines freiwilligen Fortbildungszertifikats möglich ist.

Die Fort- und Weiterbildungsbeiträge von Springer sind von den Landesärztekammern Bayern, Bremen, Hessen und Nordrhein als zertifizierte Fortbildung anerkannt. Als solche sollen sie nach einer generellen Empfehlung des Vorstands der Bundesärztekammer auch von anderen zertifizierenden Landesärztekammern anerkannt werden. Im Rahmen der Pilotphase ergibt das vollständige Ausfüllen des Fragebogens nach den einheitlichen Bewertungskriterien einen Fortbildungspunkt. Zielgruppe für das freiwillige Fortbildungszertifikat sind
Fachärzte, jedoch sollen die Fragebögen auch Ärzten in Weiterbildung zur Selbstkontrolle dienen.

\section{Den Anschluss nicht verpassen}

CME ist in aller Munde und entsprechend hoch ist die Resonanz auf unsere Initiative. Machen Sie also mit und verpassen Sie nicht den Anschluss. Dieser Service ist im Individualabonnement inbegriffen und damit für Sie kostenlos. Für Leser von Klinik- und Institutsabos sowie von Nicht-Abonnenten arbeiten wir noch an einer praktikablen Lösung wir halten Sie auf dem Laufenden.

Wir möchten, dass Sie von den Springer-Facharztzeitschriften in Ihrer Fort- und Weiterbildung maximal profitieren. Hierbei sind wir auf Ihre Fragen und Anregungen angewiesen und freuen uns über Ihren Kommentar.

Für die Redaktion Facharztzeitschriften

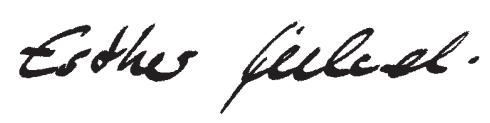

Dr. Esther Wieland

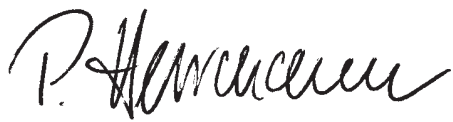

Dr. Paul Herrmann

Dr. E. Wieland, Dr.P. Herrmann

Springer-Verlag

Tiergartenstraße 17

69121 Heidelberg 